\title{
Trans-epithelial Fluid Pumping Performance of Renal Epithelial Cells and Mechanics of Cystic Expansion
}

\section{Mohammad Ikbal Choudhury}

Johns Hopkins University https://orcid.org/0000-0002-3241-9813

\section{Yizeng Li}

Johns Hopkins University

Panagiotis Mistriotis

Auburn University https://orcid.org/0000-0002-8069-3278

\section{Ana Vasconcelos}

Johns Hopkins University

\section{Eryn Dixson}

University of Maryland, Baltimore

Jing Yang

Johns Hopkins University

\section{Morgan Benson}

Johns Hopkins University

Debonil Maity

Johns Hopkins University

Rebecca Walker

Medical Research Council Harwell https://orcid.org/0000-0002-2473-4303

\section{Leigha Martin}

Johns Hopkins University

\section{Fatima Koroma}

Johns Hopkins University

\section{Feng Qian}

Johns Hopkins University

\section{Konstantinos Konstantopoulos}

Johns Hopkins University https://orcid.org/0000-0003-2623-1459

\section{Owen Woodward}

University of Maryland School of Medicine https://orcid.org/0000-0001-9514-2180

\section{Sean Sun ( $\nabla$ ssun@jhu.edu )}

$$
\text { Johns Hopkins University https://orcid.org/0000-0002-9077-7088 }
$$




\section{Research Article}

Keywords: renal cysts, fluid transport activity, kidney cells

Posted Date: May 28th, 2021

DOI: https://doi.org/10.21203/rs.3.rs-524708/v1

License: (c) (i) This work is licensed under a Creative Commons Attribution 4.0 International License. Read Full License 


\title{
Trans-epithelial Fluid Pumping Performance of Renal Epithelial Cells and Mechanics of Cystic Expansion
}

\author{
Mohammad Ikbal Choudhury ${ }^{1,3}$, Yizeng Li $i^{1,4}$, Panagiotis Mistriotis ${ }^{2,5}$, Ana C. N. Vasconcelos ${ }^{1,3}$, \\ Eryn E. Dixon ${ }^{7,8}$, Jing Yang ${ }^{1,3}$, Morgan Benson ${ }^{2}$, Debonil Maity ${ }^{2,3}$, Rebecca Walker ${ }^{6,8}$, Leigha \\ Martin $^{2}$, Fatima Koroma ${ }^{2}$, Feng Qian ${ }^{6,8}$, Konstantinos Konstantopoulos ${ }^{2,3}$, Owen M. Woodward ${ }^{7,8}$, \\ and Sean X. Sun ${ }^{1,3}$
}

${ }^{1}$ Department of Mechanical Engineering, ${ }^{2}$ Department of Chemical and Biomolecular Engineering, ${ }^{3}$ Institute of NanoBioTechnology, Johns Hopkins University, Baltimore MD USA

${ }^{4}$ Department of Mechanical Engineering, Kennesaw State University, Marietta GA USA

${ }^{5}$ Department of Chemical Engineering, Auburn University, Auburn AL USA

${ }^{6}$ Department of Biochemistry and Molecular Biology, ${ }^{7}$ Department of Physiology, ${ }^{8}$ Maryland PKD Research and Clinical Core Center, University of Maryland School of Medicine, Baltimore MD USA

\begin{abstract}
Using a novel microfluidic platform to recapitulate fluid transport activity of kidney cells, we report that renal epithelial cells can actively generate hydraulic pressure gradients across the epithelium. The fluidic flux declines with increasing hydraulic pressure until a stall pressure, at which the flux vanishes--in a manner similar to mechanical fluidic pumps. The developed pressure gradient translates to a force of 50-100 nanoNewtons per cell. For normal human kidney cells, the fluidic flux is from apical to basal, and the pressure is higher on the basal side. For human Autosomal Dominant Polycystic Kidney Disease (ADPKD) cells, the fluidic flux is reversed from basal to apical with a higher stall pressure. Molecular studies and proteomic analysis reveal that renal epithelial cells are sensitive to hydraulic pressure gradients, developing different expression profiles and spatial arrangements of ion exchangers and the cytoskeleton in different pressure conditions. These results, together with data from osmotic and pharmacological perturbations of fluidic pumping, implicate mechanical force and hydraulic pressure as important variables during morphological changes in epithelial tubules, and provide further insights into pathophysiological mechanisms underlying the development of high luminal pressure within renal cysts.
\end{abstract}


Many organs are made of a series of tubules lined with epithelial cells. For the human kidney, roughly one million nephrons with 30 kilometers of epithelial tubules re-absorb $180 \mathrm{~L}$ of water per day [1]. While the absorption activity of renal epithelial cells has been studied both in vitro and in vivo [2-4], the influence of forces and hydraulic pressures during absorption has not been examined, mainly due to difficulty in controlling these variables during experimentation. Mechanical forces are recognized as important elements during cell growth, differentiation and tissue morphogenesis [5-7]. For kidney disorders such as Autosomal Dominant Polycystic Kidney Disease (ADPKD), where tubular morphology of the epithelium becomes disrupted and uncontrolled expansion of the cyst results, mutations in the polycystin genes (PKD1 and PKD2) and their protein products (PC1 and PC2) could also alter the mechanical state of the kidney epithelium [8]. To make progress, we developed a micro-fluidic device to measure trans-epithelial fluid absorption activities of the kidney epithelium while allowing for cell imaging and simultaneous control of fluid pressure, shear stress (FSS), and media chemical composition (Fig. 1a-f and Extended Data Fig. 1a). The device measures fluidic flux across the epithelium as a function of apical and basal pressures using a microcapillary (MC) connected to port 2 (S2) or 3 (S1) (Fig. 1e,f and Fig. 3b,f). The MC simultaneously measures the trans-epithelial fluid flux and the hydraulic pressure (Fig. 1e,f) with a volume resolution of $0.31 \mu \mathrm{L}$, and can detect pressure changes of $10 \mathrm{~Pa}$. Calibration experiments were performed to obtain the pressure contribution from the MC due to capillary action and the static pressure profiles of each device for the shear flow condition considered (Extended Data Fig. 1b,c). Flow and pressure profiles of the entire device were also validated by finite element simulation (Extended Data Fig. 1d-i and SI).

We first used the well-studied MDCK-II renal cell model to characterize the device. When MDCK-II cells were seeded in the apical channel of the microfluidic device, cells settled on the porous membrane pre-treated with fibronectin and grew to confluence in 2-3 days. Upon further maturation, the epithelium showed classical cuboidal columnar morphology and formed a strong barrier, as tested using a dye permeation assay (see SI and Extended Data Fig. 3a-f). Visualization using immunofluorescence (IF) showed that F-actin, Na/K ATPase and E-cadherin were localized in typical fashion (Fig. 1k). Mature MDCK-II epithelium developed apical to basal fluid flow, which can be visualized as a rise in fluid height in the $\mathrm{MC}$ beyond the static equilibrium height (Supplementary Video 1 and Fig. $1 \mathrm{~g}$ ). The trans-epithelial fluid flux $(\mathrm{J})$ from the apical to the basal channel decreased with the hydrostatic pressure gradient $\left(\Delta P=P_{\text {basal }}-P_{\text {apical }}\right)$ across the epithelium. $\mathrm{J}$ is maximal when $\Delta \mathrm{P}=0$ (denoted as $\mathrm{J}_{0}$, or zero-pressure flux), and declined until a stall pressure (static head) of $\Delta \mathrm{P}^{*} \sim 100-250 \mathrm{~Pa}$ was reached (Fig. 1h). This flux vs pressure curve resembled the classic pump performance curve (PPC) of mechanical fluid pumps. In 
contrast, for a passive filter, the pressure needs to be higher on the apical side to generate apicalbasal flow, and the flux is zero when apical and basal pressures are equal (SI Fig, 2, Extended Data Fig. 2a,b). Therefore, kidney cells are active fluid pumps and our device can be considered as a microfluidic kidney pump (MFKP). The developed mechanical force is $30-100$ nanoNewtons per cell, and constitutes a novel force generation mechanism that is perpendicular to the epithelium. This may be a general phenomenon for absorptive or secretory epithelia that perform fluid transport. Moreover, we found that PPC changes substantially under mechanical (FSS) and hypo-osmotic gradient (OSMO) perturbations (Fig. 1g-j, Extended Data Fig. 2h,i), indicating active regulation by cells.

To validate the trans-epithelial pressure gradient measured from our device, we examined mature polarized MDCK-II monolayer on 2D impermeable substrates (glass), which formed dynamic fluid-filled domes with elevated internal hydrostatic pressure [9,10]. We measured this pressure by inserting a glass micro-needle into MDCK-II domes while monitoring the curvature of an oil-media interface in the needle (Extended Data Fig. 2c-e, SI). The dome hydraulic pressure is found to be in the same range as measured in the MFKP (Extended Data Fig. 2f,g), although in domes we were unable to simultaneously monitor the trans-epithelial fluid flux. This result, together with traction force measurements of dome pressure [11], show that MDCK-II epithelium is capable of developing hydrostatic pressure of the order of $200 \mathrm{~Pa}$ by actively pumping fluid. In contrast, a confluent layer of 3T3 fibroblast cells and immature MDCK-II epithelia were unable to develop active flux or pressure (Extended Data Fig. 2a,b). To further understand the PPC curve, we also developed a mathematical model of the PPC based on active transport of an idealized solute $([12,13]$, see SI). If the active flux for the ideal solute depends linearly on the osmotic pressure difference across the cell apical and basal surface, then the model predicts a similar PPC that shifts with changes in apical hypo-osmotic gradient, as observed in experiments (Extended Data Fig. 7a-c).

In kidney cells, $\mathrm{Na}^{+} / \mathrm{K}^{+}$ATPase (NKA) is responsible for directional $\mathrm{Na}^{+}$transport and generation of osmotic gradients and subsequent water flow, and is a major driving force for vectorial fluid transport [14]. The existence of a stall pressure could be the result of an intrinsic limit in ion pumps such as NKA, or the result of active regulation by cells in response to pressure change. NKA is polarized and accumulates in the cell baso-lateral surface. Blocking NKA by adding ouabain in the device's apical channel decreased trans-epithelial flux and stall pressure (Extended Data Fig. 4b-d). To visualize NKA dynamics in live cells, we imaged MDCK-II epithelium stably expressing SNAP-tagged NKA $\alpha$-subunit stained with TMR-STAR dye [15] while 
modulating the hydraulic pressure of the basal chamber (Fig. 2a and Supplementary Videos 3). This allowed us to visualize NKA localization in real time using confocal microscopy. Upon application of a pressure gradient $(\Delta \mathrm{P})$, NKA levels at the cell baso-lateral surface dramatically reduced over several minutes, but recovered when the applied pressure is released (Fig. 2b-d). Comparing IF images of cells in MFKP at $\Delta \mathrm{P}=0$ and $\triangle \mathrm{P}=200 \mathrm{~Pa}$ ) showed unchanged F-actin distribution, but NKA showed reduced enrichment at the baso-lateral surface when $\triangle \mathrm{P}=200 \mathrm{~Pa}$ is applied (Extended Data Fig. 4 e-n). Live cell imaging of F-actin (See SI Methods) also showed the appearance of highly dynamic F-actin invaginations at the cell basal surface with the application of $\Delta P$ (Fig. 2e and Supplementary Videos 4). The invaginations have a lifetime of $\sim 15$ seconds and eventually diminished in time when $\Delta P$ is removed (Fig. $2 \mathrm{f}, \mathrm{g}$ ). It was previously shown that disruption of cortical F-actin leads to disruption of baso-lateral NKA $[16,17]$. Therefore, we conclude that $\triangle \mathrm{P}$ causes remodeling of NKA localization, which indicates a novel mechanisms of cell pressure sensing and a negative feedback between pressure gradient and fluid flux.

For MDCK II domes on glass (Supplementary Videos 5 and 6), NKA also showed a similar reduction in enrichment on the baso-lateral domain when the dome is stable (Extended Data Fig. $40-z)$. These results indicate that cells can actively sense the apical-basal pressure difference and modulate NKA localization at the baso-lateral surface.

Next, we considered whether the device is useful for understanding fluidic pumping by primary normal human kidney cells (NHK) and ADPKD cystic cells from patients with germline PKD1 mutations. AQP2 (blue), Na/K ATPase (red) and F-actin (green) stains for cortical cells (NHKc), medullary cells (NHKm) and cystic cells (ADPKD) in MFKP showed the same distribution and morphology as those obtained from their corresponding immunohistochemistry images of kidney tissue sections (Extended Data Fig. 5a-d, f-k), suggesting that our device is capturing the general organization of these different types of epithelia. The barrier function of the epithelia was again assessed using dye permeation assay and trans-epithelial electrical resistance (TEER) (Extended Data Fig. 6a-e and SI).

Once grown in the device, as with MDCK-II epithelium, apical to basal fluid flux was observed as a function of hydrostatic pressure gradient in NHKc and NHKm epithelium, resulting in a similar PPC (Fig. 3i). $\triangle \mathrm{P}^{*}$ was higher in case of NHKc as compared to NHKm (Fig. 3j). Unlike absorptive function of normal kidney cells, ADPKD cystic cells had a secretory phenotype even though there is no dramatic difference in typical markers of apical-basal polarity $[9,18]$ (Extended Data Fig. 5a-d). The reversal of fluid flux in cystic cells remains unexplained and the underlying physical parameters of reversal have not been previously quantified. When the MC is connected 
to port 2 (S2) instead of port 3, we observed fluid rises in the MC beyond the equilibrium height by ADPKD cells, indicating basal-to-apical fluidic pumping (Fig. 3g,h, Supplementary Video 2), whereas $\mathrm{NHKm}$ and NHKc did not develop fluid flux in S2. In contrast, when the MC is connected port 3 (S1), ADPKD cells did not generate any fluid flux (Fig. 3j). In both normal and ADPKD cells, the trans-epithelial fluid flux (J) and the PPCs are modulated by mechanical (FSS) and hypoosmotic (OSMO) perturbations. These changes were quantified by plotting $\mathrm{J}_{0}$ and $\Delta \mathrm{P}^{*}$ under different conditions (Fig. 3k-n).

Under apical hypo-osmotic treatment, proximal tubule cells (NHKc) changed their pumping performance by increasing both $\mathrm{J}_{0}$ and $\Delta \mathrm{P}^{*}$. NHKm cells also increased $\mathrm{J}_{0}$ and $\Delta \mathrm{P}^{*}$ with apical hypo-osmotic shock (Fig. 3k,m). For ADPKD cells, while $\mathrm{J}_{0}$ increased with decreasing basal osmolarity, $\Delta \mathrm{P}^{*}$ didn't change and remained constant around -300 Pa (Fig. 3m). The converging nature of the PPC to a constant $\Delta \mathrm{P}^{*}$ for ADPKD epithelium (Extended Data Fig. 7f) cannot be explained by the simple active flux model (Extended Data Fig. $6 \mathrm{c}$ and $\mathrm{SI}$ ), suggesting differential mechanisms of regulation during fluidic pumping in normal and ADPKD epithelium.

In both NHKc and NHKm cells, apical FSS for 5 hours did not change $\mathrm{J}_{0}$ or $\Delta \mathrm{P}^{*}$ significantly (Fig. 3l,n). At 1 dyn $/ \mathrm{cm}^{2}$, both $\mathrm{J}_{0}$ and $\Delta \mathrm{P}^{*}$ decreased for both $\mathrm{NHKc}$ and $\mathrm{NHKm}$ as compared to control cells. However, in case of ADPKD, even though increasing apical FSS resulted in a decrease in the average $\mathrm{J}_{0}$, the fluidic flux direction reversed under FSS (Fig. 3I) with $\Delta \mathrm{P}^{*}$ going from -300 Pa to $100 \mathrm{~Pa}$ (Fig. 3n and Extended Data Fig. 7i). IF images indicate an increase in NKA localization on the lateral surface of ADPKD cells under FSS, which could potentially explain the fluidic flux reversal (Extended Data Fig. 13).

To confirm our observations from the human primary cells we next utilized an immortalized engineered mouse cell line from the Maryland PKD Cell Culture and Engineering Core with inducible Pkd2 knockout (Pkd2 $2^{f / l}$, Pax8rtTA, TetOCre $\left.[19,20]\right)$. These cells are clonal, and the inducible nature of the $\mathrm{KO}$ provides isogenic controls with low genotypic and phenotypic noise, allowing for mechanistic insights into ADPKD [19,20]. These cells also formed a tight epithelium in our device, and we measured their PPC before and after the application of doxycycline that triggered depletion of PC2 levels (Fig. 3o). Results show that $P k d 2 \mathrm{KO}$ cells lowered apical-basal flux, but did not reverse as seen in ADPKD cells (Fig. 3p-r). Noticeably, baso-lateral localization of F-actin and NKA changed dramatically when PC2 was completely depleted, which could explain the decrease in apical-basal fluid flux (Extended data Fig. 9c). The inducible Pkd2 KO cell model of ADPKD provides mechanistic insight into the most acute changes after the loss of 
polycystin proteins and the first steps of cystogenesis [18]; observed phenotypical differences with the human ADPKD mature cysts cells suggests that further transformation may occur after PC2 loss and ADPKD cells from mature cysts behave differently.

We have shown that a hydrostatic pressure gradient $(\Delta \mathrm{P})$ is developed during fluid pumping, and the cyst wall can sustain a pressure of up to $300 \mathrm{~Pa}$. In situ, this pressure points from the lumen towards the interstitium, and could drive mechanical and proliferative cyst expansion. The regulation of $\Delta \mathrm{P}^{*}$ is therefore potentially critical for understanding kidney morphogenesis. In ADPKD kidneys, the progressive growth of individual fluid filled cysts leads to a collective increase in total kidney volume (TKV) [21]. Currently, the only Food and Drug Administration (FDA) approved pharmacological treatment that decreases TKV is Tolvaptan (TVP) [21,22], a V2R antagonist and has been shown to decrease cAMP levels [23]. We treated mature ADPKD epithelium in MFKP with $1 \mathrm{nM}$ TVP on the baso-lateral side for 1 hour and measured PPC. Interestingly, TVP decreased both $\mathrm{J}_{0}$ and $\triangle \mathrm{P}^{*}$ of the ADPKD epithelium as compared to the control (Extended Data Fig. 7j), confirming that the cAMP pathway can modulate fluid flux and $\Delta \mathrm{P}^{*}$. To further confirm that human kidney cells are sensitive to pressure changes as seen in MDCK II (Fig. 2), and to obtain a molecular profile of kidney cells during fluidic pumping, we performed qPCR measurements for aquaporins (AQP1, AQP3 and AQP5), ion-pumps and exchangers (Na/K ATPase, NHE1, NKCC1, NKCC2, CFTR), and tension sensitive $\mathrm{Ca}^{2+}$ channels (TRPM7 and TRPV4), which are all potentially involved in regulating absorptive and secretory water/ion transport, and mechano-sensation. Heatmaps indicating the expression of mRNAs extracted from NHKc, NHKm and ADPKD cells grown on permeable and impermeable substrate (tissue culture treated polystyrene dishes) show substantial expression differences (Extended Data Fig. 8a). Moreover, we collected cells from MFKP under two conditions: $\Delta P=0(C T)$ and $\Delta P$ $=200 \mathrm{~Pa}$. Fig. 4b,d show that when exposed to pressure $\Delta \mathrm{P}=200 \mathrm{~Pa}, \mathrm{NHKm}$ cells decreased the expressions of genes, particularly ATP1A1 (NKA). However, ADPKD cells did not respond to $\triangle P$, where expressions of these genes either remained constant or increased slightly. Mouse $P k d 2 \mathrm{KO}$ cells also showed substantial expression change similar to human cells when exposed to $\Delta \mathrm{P}=200 \mathrm{~Pa}$ as compared to DMSO control (Fig. 4e,f). Interestingly, diseased cells (both mouse and human) did not respond to $\Delta P$ as normal cells, particularly the mRNA levels of ATP1A1 (NKA) (green rectangle) (Fig. 4b,d,e,f). IF images of F-actin and NKA in the MFKP device corroborates the $\mathrm{qPCR}$ results at $\triangle \mathrm{P}=0$ and $\triangle \mathrm{P}=200 \mathrm{~Pa}$. The total intensities of NKA in NHKc, $\mathrm{NHKm}$ and ADPKD epithelia under the two conditions were also consistent with the mRNA readings from qPCR (Extended Data Fig. 10-12 and Fig. 4a,c). However, spatial arrangement of F-actin and NKA in NHKc, NHKm and ADPKD epithelia showed significant differences at $\triangle P=0$ 
and $\Delta \mathrm{P}=200 \mathrm{~Pa}$ ). For all the three human primary cell types, F-actin was generally the highest at the cell basal surface at $\Delta P=0$, but the F-actin stress fiber density was significantly decreased at $\Delta \mathrm{P}=200 \mathrm{~Pa}$ (Extended Data Fig. 10-12). Interestingly, $\Delta \mathrm{P}$ disrupted the baso-lateral polarization of NKA in NHKm cells (Extended Data Fig. 11a-r). This depolarization effect was completely absent in NHKc but was subtle in ADPKD cells exposed to $\triangle \mathrm{P}$. This indicates that collecting duct cells (NHKm population) have altered pressure sensing properties than NHKc and ADPKD cells.

Our combined results offer insights into kidney fluidic pumping action and the mechanobiology of ADPKD cyst formation. Fig. $4 \mathrm{~g}$ shows a section of a normal human kidney tubule. Blue arrows represent fluidic flux from the lumen into the interstitial space. Black arrows indicate the restoring force as a result of the fluidic pumping, arising from the apical-basal hydraulic pressure difference during pumping. Here the apical-basal pressure difference can prevent tubule expansion. During cyst initiation, due to altered morphology of the tubule, the local fluid shear stress can be lower, which may lead to reversed direction of fluid pumping by ADPKD cells (Fig. 4h). This changes the direction of the restoring force, which further destabilizes the tubule. Fluid secretion together with aberrant pressure sensing response of ADPKD cells act together to increase cyst outward pressure. Such mechanical factors coupled with cell proliferation [24] can lead to gradual cyst expansion (Fig. 4i). Our results demonstrate that secretory and absorptive functions of epithelia can generate significant mechanical forces, and these forces may be important generally in tubular morphogenesis. Our microfluidic device is a unique platform for studying epithelial cell dynamics under controlled pressure and flow conditions that can lead to novel insights.

\section{Acknowledgements}

The authors would like to thank Dr. Michael J. Caplan Lab for providing the SNAP-tagged MDCKII cell line. The work has been supported by NIH R01GM114674 and a pilot grant from Maryland PKD Research and Clinical Core Center. 


\section{References}

1. Taal, M., Chertow, G., Marsden, P., Skorecki, K., Yu, A. \& Brenner B. (2011). The Kidney, $9^{\text {th }}$ Ed.

2. Weinstein, A. M. (2000). Sodium and chloride transport: proximal nephron. The kidney. Physiology and pathophysiology.

3. Sullivan, L. P., Wallace, D. P., \& Grantham, J. J. (1998). Epithelial transport in polycystic kidney disease. Physiological reviews.

4. Burg, M. B., \& Orloff, J. (1968). Control of fluid absorption in the renal proximal tubule. The Journal of clinical investigation, 47(9), 2016-2024.

5. Dasgupta, S., Gupta, K., Zhang, Y., Viasnoff, V., \& Prost, J. (2018). Physics of lumen growth. Proceedings of the National Academy of Sciences, 115(21), E4751-E4757.

6. Heisenberg, C. P., \& Bellaïche, Y. (2013). Forces in tissue morphogenesis and patterning. Cell, 153(5), 948-962.

7. Miroshnikova, Y. A., Le, H. Q., Schneider, D., Thalheim, T., Rübsam, M., Bremicker, N., ... \& Balland, M. (2018). Adhesion forces and cortical tension couple cell proliferation and differentiation to drive epidermal stratification. Nature cell biology, 20(1), 69.

8. Torres, V. E., \& Harris, P. C. (2006). Mechanisms of disease: autosomal dominant and recessive polycystic kidney diseases. Nature Reviews Nephrology, 2(1), 40.

9. Lever, J. E. (1979). Inducers of mammalian cell differentiation stimulate dome formation in a differentiated kidney epithelial cell line (MDCK). Proceedings of the National Academy of Sciences, 76(3), 1323-1327.

10. Yang, J., Duan, X., Fraser, A. K., Choudhury, M. I., Ewald, A. J., Li, R., \& Sun, S. X. (2019). Microscale pressure measurements based on an immiscible fluid/fluid interface. Scientific Reports, $9,1-10$.

11. Latorre, E., Kale, S., Casares, L., Gómez-González, M., Uroz, M., Valon, L., ... \& Trepat, X. (2018). Active superelasticity in three-dimensional epithelia of controlled shape. Nature, 563(7730), 203208.

12. Stroka, K. M., Jiang, H., Chen, S. H., Tong, Z., Wirtz, D., Sun, S. X., \& Konstantopoulos, K. (2014). Water permeation drives tumor cell migration in confined microenvironments. Cell, 157(3), 611 623.

13. Li, Y., Mori, Y., \& Sun, S. X. (2015). Flow-driven cell migration under external electric fields. Physical review letters, 115(26), 268101.

14. Kennedy, B. G., \& Lever, J. E. (1984). Regulation of Na+, K+-ATPase activity in MDCK kidney epithelial cell cultures: Role of growth state, cyclic AMP, and chemical inducers of dome formation and differentiation. Journal of cellular physiology, 121(1), 51-63. 
15. Farr, G. A., Hull, M., Mellman, I., \& Caplan, M. J. (2009). Membrane proteins follow multiple pathways to the basolateral cell surface in polarized epithelial cells. Journal of Cell Biology, 186(2), 269-282.

16. Nelson, W. J., \& Veshnock, P. J. (1987). Ankyrin binding to (Na++ K+) ATPase and implications for the organization of membrane domains in polarized cells. Nature, 328(6130), 533-536

17. Duan, Y., Weinstein, A. M., Weinbaum, S., \& Wang, T. (2010). Shear stress-induced changes of membrane transporter localization and expression in mouse proximal tubule cells. Proceedings of the National Academy of Sciences, 107(50), 21860-21865.

18. Wilson, P. D. (2004). Polycystic kidney disease. New England Journal of Medicine, 350(2), 151164.

19. Dixon, E. E., Maxim, D. S., Kuhns, V. L. H., Lane-Harris, A. C., Outeda, P., Ewald, A. J., ... \& Woodward, O. M. (2020). GDNF drives rapid tubule morphogenesis in a novel 3D in vitro model for ADPKD. Journal of cell science, 133(14).

20. Jung, H. J., Coleman, R. A., Dixon, E. E., Outeda, P. O., Woodward, O. M. and Welling, P. A. (2019) Identification of cystogenic signaling pathways in a newly developed, inducible - kidney epithelial cell model of Pkd2 - mediated PKD. [Abstract]. J Am Soc Nephrol 30, 2019: Page(s) 632633.

21. Grantham, J. J., \& Torres, V. E. (2016). The importance of total kidney volume in evaluating progression of polycystic kidney disease. Nature Reviews Nephrology, 12(11), 667.

22. Torres, V. E., Chapman, A. B., Devuyst, O., Gansevoort, R. T., Grantham, J. J., Higashihara, E., ... \& Czerwiec, F. S. (2012). Tolvaptan in patients with autosomal dominant polycystic kidney disease. New England Journal of Medicine, 367(25), 2407-2418.

23. Gattone II, V. H., Wang, X., Harris, P. C., \& Torres, V. E. (2003). Inhibition of renal cystic disease development and progression by a vasopressin V2 receptor antagonist. Nature medicine, $9(10)$, 1323.

24. Gudipaty, S. A., Lindblom, J., Loftus, P. D., Redd, M. J., Edes, K., Davey, C. F., ... \& Rosenblatt, J. (2017). Mechanical stretch triggers rapid epithelial cell division through Piezo1. Nature, 543(7643), 118. 


\section{Main Figure captions:}

\section{Figure 1: Fluid pumping performance of MDCK-II epithelium.}

(a) A schematic representation of the Micro-fluidic Kidney Pump (MFKP). (b) A DIC image of the MDCK-II monolayer on porous polycarbonate membrane with pores size of $1 \mathrm{~mm}$. White dots indicate pores. Scale bar $=10 \mathrm{~mm}$. (c) Longitudinal section of the device with four ports: 1 and 2 are used to access the apical channel and 0 and 3 are used to access the basal channel. Black arrows are the direction of fluid flux. Port 0 is closed except during seeding and cell culture. (d) A schematic of a polarized epithelium. $P_{\text {apical }}$ and $P_{\text {basal }}$ indicate hydrostatic pressures in the apical and basal channels, respectively and arrows (blue) indicate the direction of trans-epithelial fluid flow. (e) Dashed rectangle shows zoomed schematic of fluid flow in the microcapillary (MC) and a mm scale (green). The MC measures both trans-epithelial fluid flux $(\mathrm{J})$ and hydrostatic pressure gradient (DP). (f) A snapshot of the MFKP inside an incubator for time-lapsed videography. The dashed line indicates MC, and 0,1, 2 and 3 label the ports. Scale bar $=1 \mathrm{~cm}$. (g) MDCK-II cells grown in MFKP generate a hydrostatic pressure gradient by pumping fluid from the apical to basal side. The height of fluid in the MC is plotted as function of time for MDCK-Il epithelium in MFKP. Compared to static condition (CT, red) $(n=11, N=3$, devices, biological replicates), the fluid pumping action changed when the apical medium is switched to $20 \%$ hypo-osmotic condition (OSMO, green) ( $n=11, N=3)$. Shaded area is the standard error of the mean (SEM). (h) Pump performance curve (PPC) of MDCK-II epithelium, showing the measured trans-epithelial fluid flux (J) (apical to basal) as a function of the hydrostatic pressure gradient ( $\left.D P=P_{\text {basal }}-P_{\text {apical }}\right)$. $J_{0}$ is the fluid flux at zero pressure gradient $(D P=0)$ and $D P^{*}$ is the stall pressure when $\mathrm{J}=0$. Both $\mathrm{J}_{0}$ and $\mathrm{DP}^{*}$ change in the hypo-osmotic condition. Shaded area is the SEM. Comparison of $\mathrm{J}_{0}$ (i) and $D P^{*}$ (j) for MDCK-II epithelium in the static condition (CT, red) $(n=11, N=3)$ and $20 \%$ apical hypo-osmotic condition (OSMO, green) ( $n=11, N=3$ ). ${ }^{* * *} p<0.0001$ and ${ }^{* * *} p<0.001$ (two-tailed Mann-Whitney t-test). (k) Immunofluorescence (IF) images showing nuclei (blue), F-actin (green), NKA (red) and E-cadherin (purple) in MDCK-II epithelium in the MFKP. The dashed line (white) in the $X Z$ panel indicates the porous membrane. The intensity projection for $X Z$ images was recorded along the corresponding dashed lines (yellow) in the XY images. Scale bar $=25 \mathrm{~mm}$. 
Figure 2: DP decreases baso-lateral $\mathrm{Na}^{+} / \mathrm{K}^{+}$ATPase localization by disrupting F-actin cortex.

(a) Live cell imaging MDCK-Il cells stably expressing SNAP-tagged $\mathrm{Na}^{+} / \mathrm{K}^{+}$ATPase (NKA) asubunit and stained with TMR-STAR dye in the MFKP. DP $=0$ indicates zero hydrostatic pressure gradient (i.e., $\mathrm{P}_{\text {apical }}=\mathrm{P}_{\text {basal }}$ ) and $\mathrm{DP}=200 \mathrm{~Pa}$ indicates a hydrostatic pressure gradient of $200 \mathrm{~Pa}$ (i.e., $\mathrm{P}_{\text {basal }}=\mathrm{P}_{\text {apical }}+200 \mathrm{~Pa}$ ). Time-lapsed confocal images of cells' lateral side in three consecutive conditions: zero pressure $(\mathrm{DP}=0)$, pressure gradient $(\mathrm{DP}=200 \mathrm{~Pa})$ and pressure released $(D P=0)$. The arrowhead indicates disruption of lateral NKA expression in regions of interest (ROls) at various time stamps. Scale bar $=10 \mathrm{~mm}$. (b) A zoomed confocal slice of an ROI in MDCK-II cells expressing SNAP-tagged NKA and stained with TMR-STAR dye. (c) NKA intensity is quantified in a band (red box) centered between two cells in $\mathbf{b}$. The intensity profile in $\mathbf{c}$ is the vertical average intensity along the red box in $\mathbf{b}$, the peak (indicated by black arrow) is the maximum intensity of NKA. (d) Mean of normalized peak NKA intensity on the lateral side for the same cell over the course of three pressure conditions in a. Shading represents the SEM. ( $n$ $=25, \mathrm{~N}=3$, cells, biological replicates). The green region indicates $\mathrm{DP}=200 \mathrm{~Pa}$. (e) Live cell imaging of transiently transfected MDCK-II cells expressing GFP-tagged F-actin (Ftractin). Timelapsed confocal images of the baso-lateral side of the cells were taken under three consecutive conditions: $\mathrm{DP}=0, \mathrm{DP}=200 \mathrm{~Pa}$, and pressure released $(\mathrm{DP}=0)$. Scale bar $=10 \mathrm{~mm}$. Pressure application induces rapid high-frequency invaginations in the baso-lateral domain. White arrowhead indicates F-actin rings, which are cross-section of invaginations. (f) Number of invaginations per unit cell over the course of three pressure conditions in $\mathbf{e}(n=8, N=3$, cells, biological replicates). (g) Mean area of invaginations as a function of time under pressure. Shading represents the SEM. ( $n=10, N=3$, cells, biological replicates). The invaginations have an average lifetime of $\sim 15$ seconds and disappear when pressure is released as shown in $\mathbf{f}$. (h) Schematic showing co-localization of F-actin (green lines) and NKA (red dots) in cells in MFKP at $\mathrm{DP}=0$. (i) Hydrostatic pressure gradient results in high-frequency invaginations on the basolateral F-actin cortex and a reduction in NKA localization in the lateral domain. 
Figure 3: Cystic cells derived from ADPKD patients pump fluid in the opposite direction as normal human kidney cells

(a) A snapshot of MFKP in setup-2 (S2) where the microcapillary (MC) is connected to port 2. Port 1 is closed. (b) $\mathrm{S} 2$ allows the measurement of trans-epithelial fluid flux from the basal to apical chamber. (c) A cystic kidney derived from ADPKD patients. (d) A normal kidney derived from patients. (e) A snapshot of the MFKP in setup-1 (S1) where the MC is connected to port 3 in the MFKP. Port 1 is closed. (f) Schematic of $\mathbf{S} 1$ which enables measurement of trans-epithelial fluid flux from apical to basal chamber. (g) Comparison of zero-pressure flux, $J_{0}$, and stall pressure, DP*, for NHKc, NHKm and ADPKD epithelia in MFKP measured using S2. (h) Pump performance curve (PPC) of ADPKD epithelium grown in MFKP using S2. ADPKD cystic cells pump fluid from basal to apical side and the trans-epithelial fluid flux $(\mathrm{J})$ decreases with increasing DP across the epithelium. ( $\left.D P=\mathrm{P}_{\text {basal }} \mathrm{P}_{\text {apical }}\right)$. ( $\mathrm{N}=13$ biological replicates). (i) PPCs of NHKc ( $N$ $=10)$ and $\mathrm{NHKm}(\mathrm{N}=8)$ epithelia in MFKP using $\mathrm{S} 1$. (j) Comparison of zero-pressure flux, $\mathrm{J}_{0}$, and stall pressure, DP*, for NHKc, NHKm and ADPKD epithelium in MFKP measured using S1. Comparisons of $\mathrm{J}_{0}$ in NHKc, NHKm and ADPKD epithelia with varying hypo-osmotic gradients (k) and fluid shear stresses (I). Comparison of $\mathrm{DP}^{*}$ in NHKc, NHKm and ADPKD epithelia with varying hypo-osmotic gradients $(\mathrm{m})$ and fluid shear stresses $(\mathbf{n})$. (o) Protocol for genetic knock-out in a $P k d 2^{f / f f l}$, Pax8rtTA, TetOCre mouse cell line. Western blot showing depletion of polycystin-2 protein abundance in doxycycline (Dox) treated cells compared to DMSO controls. (p) PPCs for normal (red) ( $n=22, N=3$ ) vs $P k d 2 \mathrm{KO}$ (blue) ( $\mathrm{n}=27, \mathrm{~N}=3$, devices, biological repeats) mouse kidney epithelium in MFKP using S1. Comparison of zero-pressure flux, $\mathrm{J}_{0}$, (q) and stall pressure, $\mathrm{DP}^{*}$, (r) for normal vs. Pkd2 KO mouse kidney epithelium measured using $\mathrm{S} 1 .{ }^{*} \mathrm{p}<0.05,{ }^{* *} \mathrm{p}<$ $0.01,{ }^{* * *} p<0.001,{ }^{* * *} p<0.0001$. Unpaired, two-tailed, standard t-test).

Figure 4: Diseased cells have defects in sensing hydrostatic pressure as compared to normal cells

(a) Comparison of total $\mathrm{Na}^{+} / \mathrm{K}^{+}$ATPase (NKA) expression in human NHKm cells and ADPKD cystic cells using antibody staining of the $\mathrm{Na} / \mathrm{K}$ ATPase a-subunit. Maximum intensity projections of confocal stacks were used to quantify NKA expression. The epithelium was studied under two conditions: CT (Control, DP = 0) and DP (hydrostatic pressure gradient, DP = 200 Pa for 5 hours). Plot showing total intensity of NKA in NHKc cells at CT and DP. $(n=20, N=3)$. (b) Comparison 
of relative expression changes of genes in NHKm cells for CT (DP=0) and DP (DP = $200 \mathrm{~Pa})$. ( $\mathrm{n}$ $=9, N=3)$. (c) Plot showing total intensity of NKA in human ADPKD cystic cells for CT $(D P=0)$ and DP = -200 Pa ( $\mathrm{ns}=$ not significant, $\mathrm{p}=0.74),(\mathrm{n}=20, \mathrm{~N}=3)$. (d) Comparison of gene expression changes in human ADPKD cystic cells for CT $(D P=0)$ and $D P=-200 P a(n=9, N=$ 3). Green rectangle indicates the comparison for ATP1A1 (which encodes for NKA). For human NHKm cells, mRNA levels of ATP1A1 decreased significantly ( $\left.{ }^{* * *} p \$<0.001 \$\right)$ under hydrostatic pressure for 10 hours but remained unchanged in case of cystic cells ( $p=0.5170)$. (e) Comparison of relative expression changes of genes in inducible mouse cells ( $P k d 2^{f / f t}$, Pax8rtTA, TetOCre) with DMSO vehicle for CT (DP = 0) and DP = $200 \mathrm{~Pa}(\mathrm{n}=6, \mathrm{~N}=2)$. (f) Comparison of relative expression changes of genes in doxycycline induced $P k d 2 \mathrm{KO}$ mouse cells for CT $(\mathrm{DP}=0)$ and $\mathrm{DP}=200 \mathrm{~Pa} .(\mathrm{n}=12, \mathrm{~N}=4)$. For normal mouse cells, the mRNA levels of ATP1A1 gene increased significantly $\left({ }^{* * * *} p<0.0001\right)$ under hydrostatic pressure for 10 hours but remained unchanged in case of Pkd2 KO cells $(p=0.4856)$. Error bars indicate SEM. $\left({ }^{*} p<0.05,{ }^{* *} p<0.01,{ }^{* * *} p<0.001\right.$, ${ }^{* * *} p<0.0001$. Unpaired, two-tailed, standard t-test). (g) A schematic representation of a tubular nephron section in a normal kidney. The blue arrows indicate absorptive fluid flux from the lumen into the interstitial space. The black arrows indicate the pumping force generated by cells. The tubular structure is stabilized by the pumping force due to apico-basal fluid flux (radially in). (h) During cyst initiation, cell proliferation can alter the tubule geometry and the local fluid flow pattern, results in a lowered FSS. ADPKD cells may respond by reversing the fluidic pumping direction as shown in Fig. 3o, which also reverses the pumping force that further destabilizes the tubule. (i) In mature cysts, fluid pumping elevates pressure in the cyst and aids in cyst expansion. 


\section{Figures}

a

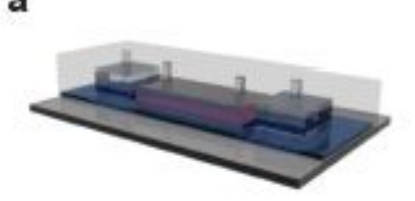

d

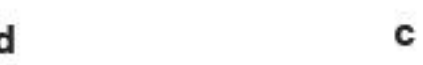

b

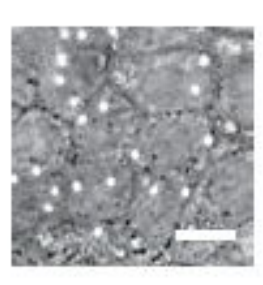

f

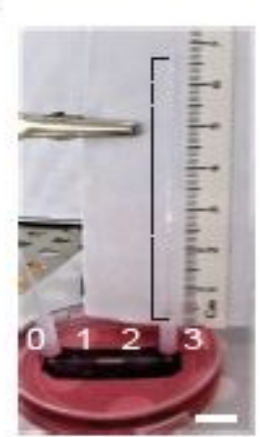

j g

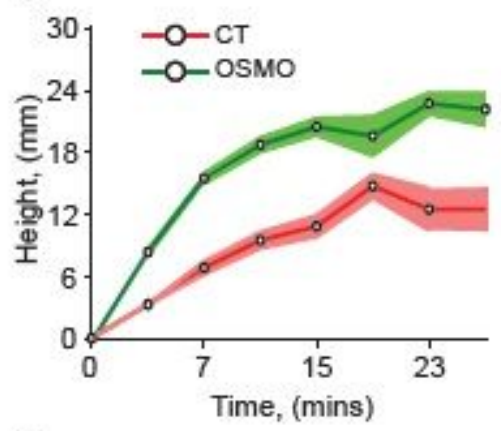

h

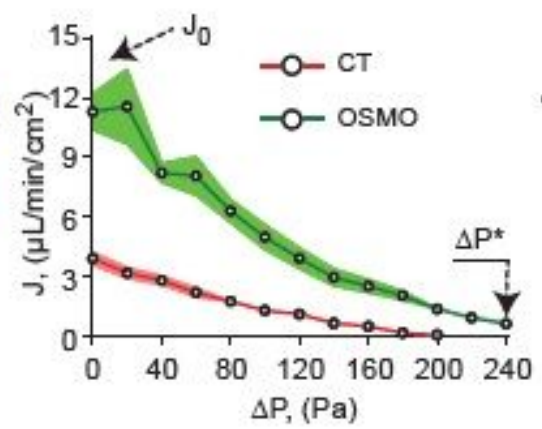

i

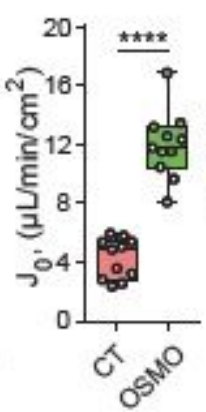

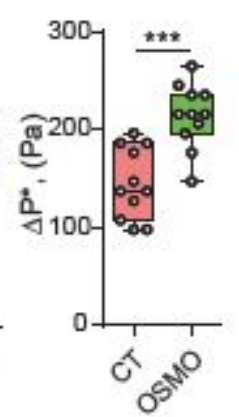

k
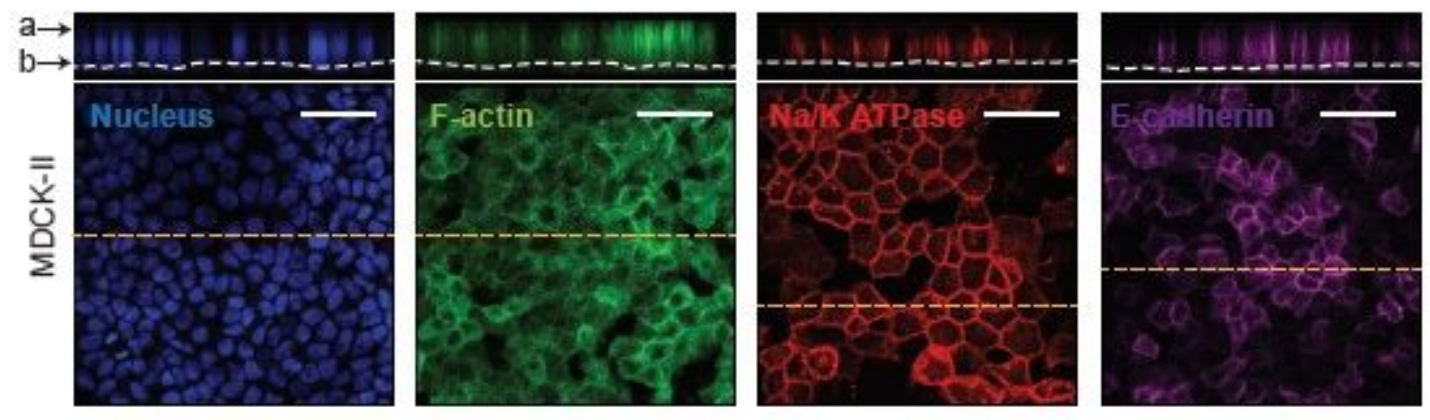

\section{Figure 1}

Fluid pumping performance of MDCK-II epithelium. (a) A schematic representation of the Micro-fluidic Kidney Pump (MFKP). (b) A DIC image of the MDCK-II monolayer on porous polycarbonate membrane with pores size of $1 \mathrm{~mm}$. White dots indicate pores. Scale bar $=10 \mathrm{~mm}$. (c) Longitudinal section of the device with four ports: 1 and 2 are used to access the apical channel and 0 and 3 are used to access the basal channel. Black arrows are the direction of fluid flux. Port 0 is closed except during seeding and cell culture. (d) A schematic of a polarized epithelium. Papical and Pbasal indicate hydrostatic pressures in the apical and basal channels, respectively and arrows (blue) indicate the direction of trans-epithelial fluid flow. (e) Dashed rectangle shows zoomed schematic of fluid flow in the microcapillary (MC) and a mm scale (green). The MC measures both trans-epithelial fluid flux (J) and hydrostatic pressure gradient (DP). (f) A snapshot of the MFKP inside an incubator for time-lapsed videography. The dashed line indicates 
MC, and 0,1, 2 and 3 label the ports. Scale bar $=1 \mathrm{~cm}$. (g) MDCK-II cells grown in MFKP generate a hydrostatic pressure gradient by pumping fluid from the apical to basal side. The height of fluid in the MC is plotted as function of time for MDCK-II epithelium in MFKP. Compared to static condition (CT, red) ( $\mathrm{n}=$ $11, N=3$, devices, biological replicates), the fluid pumping action changed when the apical medium is switched to $20 \%$ hypo-osmotic condition (OSMO, green) $(n=11, N=3)$. Shaded area is the standard error of the mean (SEM). (h) Pump performance curve (PPC) of MDCK-Il epithelium, showing the measured trans-epithelial fluid flux (J) (apical to basal) as a function of the hydrostatic pressure gradient (DP = Pbasal-Papical). J0 is the fluid flux at zero pressure gradient $(D P=0)$ and $D P^{*}$ is the stall pressure when $\mathrm{J}=0$. Both $\mathrm{JO}$ and $\mathrm{DP}^{*}$ change in the hypo-osmotic condition. Shaded area is the SEM. Comparison of J0 (i) and $D P^{*}(j)$ for MDCK-II epithelium in the static condition (CT, red) $(n=11, N=3)$ and $20 \%$ apical hypoosmotic condition (OSMO, green) $(n=11, N=3)$. ${ }^{\star \star *} \mathrm{p}<0.0001$ and $* \star \star p<0.001$ (two-tailed Mann-Whitney t-test). (k) Immunofluorescence (IF) images showing nuclei (blue), F-actin (green), NKA (red) and Ecadherin (purple) in MDCK-II epithelium in the MFKP. The dashed line (white) in the XZ panel indicates the porous membrane. The intensity projection for $X Z$ images was recorded along the corresponding dashed lines (yellow) in the XY images. Scale bar $=25 \mathrm{~mm}$. 


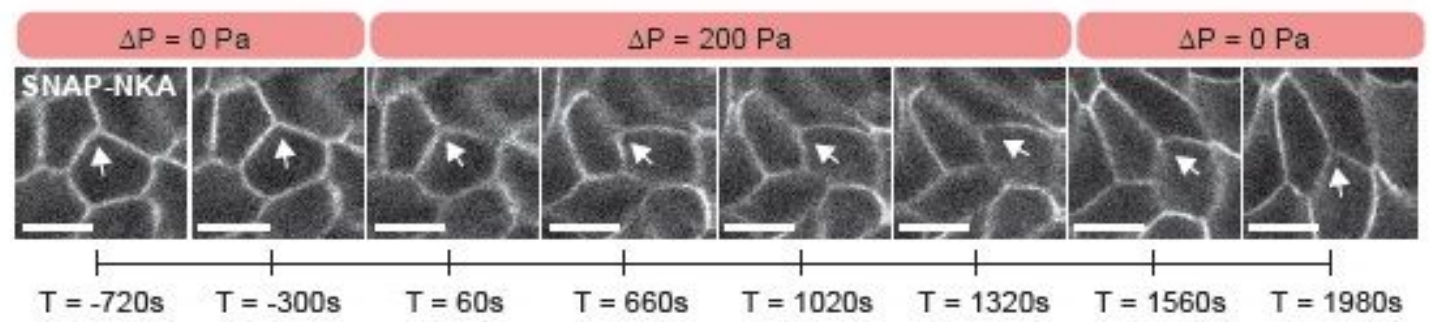

b

c

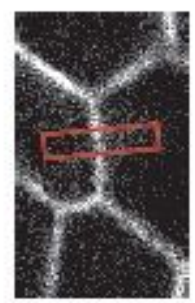

d

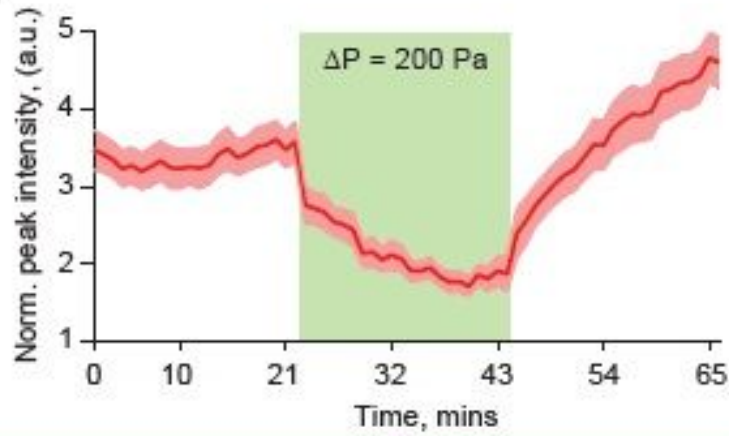

e

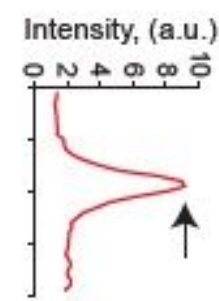

ime, mins
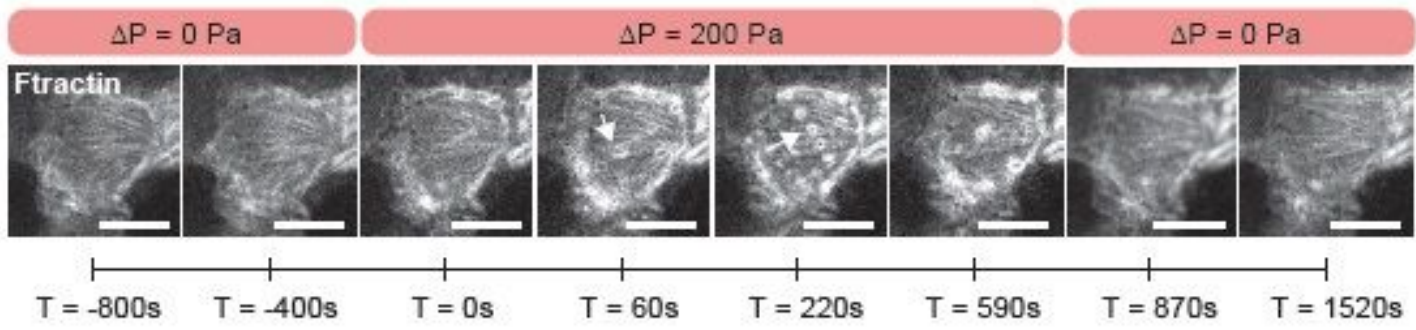

f

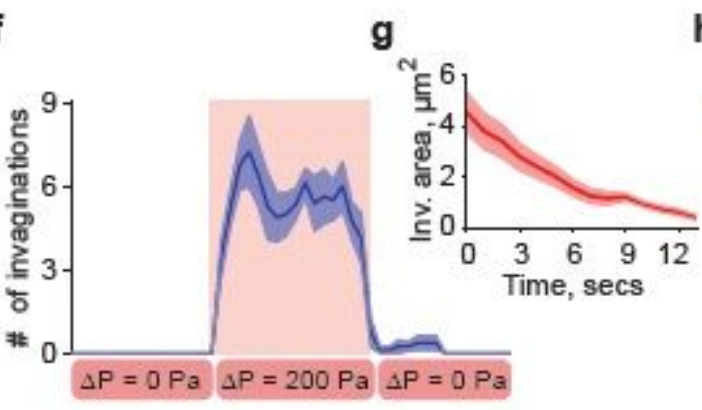

h $\quad$ i

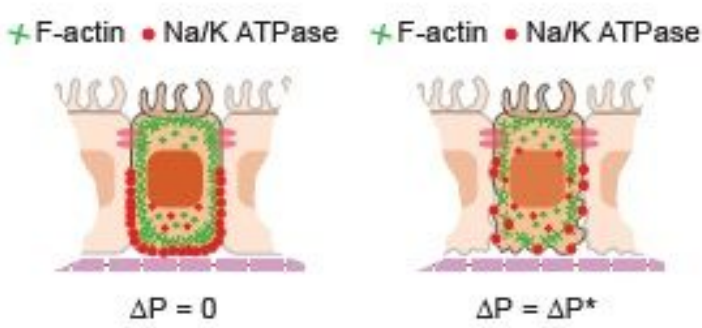

Figure 2

DP decreases baso-lateral Na+/K+ ATPase localization by disrupting F-actin cortex. (a) Live cell imaging MDCK-II cells stably expressing SNAP-tagged Na+/K+ ATPase (NKA) asubunit and stained with TMRSTAR dye in the MFKP. DP = 0 indicates zero hydrostatic pressure gradient (i.e., Papical = Pbasal) and DP = $200 \mathrm{~Pa}$ indicates a hydrostatic pressure gradient of $200 \mathrm{~Pa}$ (i.e., Pbasal $=$ Papical $+200 \mathrm{~Pa}$ ). Timelapsed confocal images of cells' lateral side in three consecutive conditions: zero pressure $(D P=0)$, pressure gradient $(\mathrm{DP}=200 \mathrm{~Pa})$ and pressure released $(\mathrm{DP}=0)$. The arrowhead indicates disruption of lateral NKA expression in regions of interest (ROIs) at various time stamps. Scale bar $=10 \mathrm{~mm}$. (b) $\mathrm{A}$ zoomed confocal slice of an ROI in MDCK-II cells expressing SNAP-tagged NKA and stained with TMRSTAR dye. (c) NKA intensity is quantified in a band (red box) centered between two cells in b. The intensity profile in $\mathrm{c}$ is the vertical average intensity along the red box in $\mathrm{b}$, the peak (indicated by black 
arrow) is the maximum intensity of NKA. (d) Mean of normalized peak NKA intensity on the lateral side for the same cell over the course of three pressure conditions in a. Shading represents the SEM. $(n=25, N$ $=3$, cells, biological replicates). The green region indicates DP $=200 \mathrm{~Pa}$. (e) Live cell imaging of transiently transfected MDCK-II cells expressing GFP-tagged F-actin (Ftractin). Timelapsed confocal images of the baso-lateral side of the cells were taken under three consecutive conditions: $D P=0, D P=$ $200 \mathrm{~Pa}$, and pressure released $(\mathrm{DP}=0)$. Scale bar $=10 \mathrm{~mm}$. Pressure application induces rapid highfrequency invaginations in the baso-lateral domain. White arrowhead indicates F-actin rings, which are cross-section of invaginations. (f) Number of invaginations per unit cell over the course of three pressure conditions in e ( $n=8, N=3$, cells, biological replicates). (g) Mean area of invaginations as a function of time under pressure. Shading represents the SEM. ( $n=10, N=3$, cells, biological replicates). The invaginations have an average lifetime of $\sim 15$ seconds and disappear when pressure is released as shown in $\mathrm{f}$. (h) Schematic showing co-localization of F-actin (green lines) and NKA (red dots) in cells in $M F K P$ at $D P=0$. (i) Hydrostatic pressure gradient results in high-frequency invaginations on the basolateral F-actin cortex and a reduction in NKA localization in the lateral domain. 


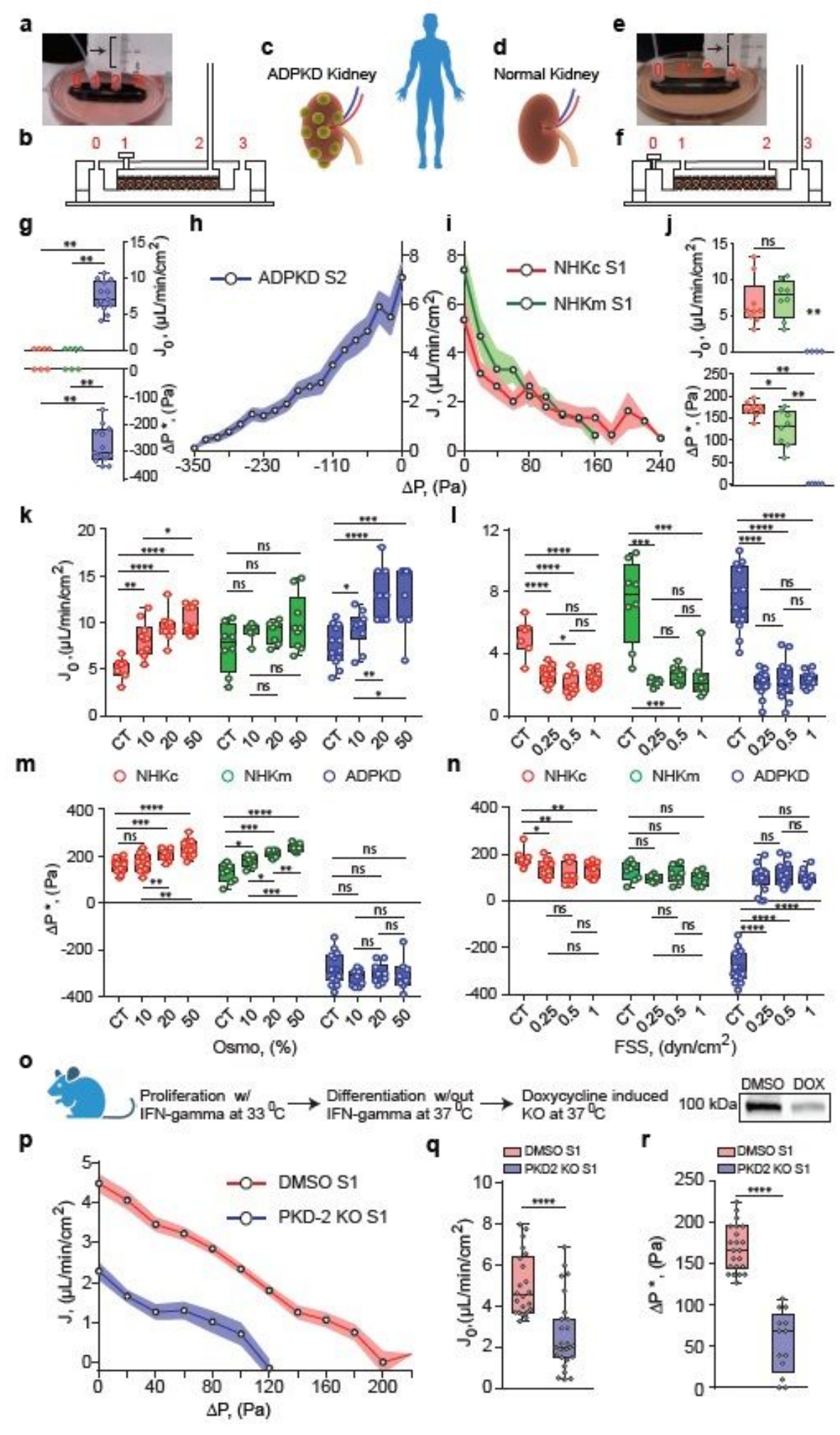

\section{Figure 3}

Cystic cells derived from ADPKD patients pump fluid in the opposite direction as normal human kidney cells (a) A snapshot of MFKP in setup-2 (S2) where the microcapillary (MC) is connected to port 2. Port 1 is closed. (b) S2 allows the measurement of trans-epithelial fluid flux from the basal to apical chamber. (c) A cystic kidney derived from ADPKD patients. (d) A normal kidney derived from patients. (e) A snapshot of the MFKP in setup-1 (S1) where the MC is connected to port 3 in the MFKP. Port 1 is closed. 
(f) Schematic of S1 which enables measurement of trans-epithelial fluid flux from apical to basal chamber. (g) Comparison of zero-pressure flux, J0, and stall pressure, DP*, for NHKc, NHKm and ADPKD epithelia in MFKP measured using S2. (h) Pump performance curve (PPC) of ADPKD epithelium grown in MFKP using S2. ADPKD cystic cells pump fluid from basal to apical side and the trans-epithelial fluid flux (J) decreases with increasing DP across the epithelium. (DP = Pbasal-Papical). ( $N=13$ biological replicates). (i) PPCs of NHKc ( $N=10)$ and NHKm ( $=8)$ epithelia in MFKP using S1. (j) Comparison of zero-pressure flux, J0, and stall pressure, DP*, for NHKc, NHKm and ADPKD epithelium in MFKP measured using S1. Comparisons of $\mathrm{J0}$ in NHKc, NHKm and ADPKD epithelia with varying hypo-osmotic gradients (k) and fluid shear stresses (I). Comparison of DP* in NHKc, NHKm and ADPKD epithelia with varying hypo-osmotic gradients $(\mathrm{m})$ and fluid shear stresses $(n)$. (o) Protocol for genetic knock-out in a $\mathrm{Pkd} 2 \mathrm{fl} / \mathrm{fl}, \mathrm{Pax} 8 \mathrm{rtTA}$, TetOCre mouse cell line. Western blot showing depletion of polycystin-2 protein abundance in doxycycline (Dox) treated cells compared to DMSO controls. (p) PPCs for normal (red) ( $\mathrm{n}=$ $22, N=3$ ) vs Pkd2 KO (blue) ( $n=27, N=3$, devices, biological repeats) mouse kidney epithelium in MFKP using S1. Comparison of zero-pressure flux, J0, (q) and stall pressure, DP*, (r) for normal vs. Pkd2 KO mouse kidney epithelium measured using $S 1$. ${ }^{*} p<0.05$, ${ }^{*} p<0.01$, ${ }^{\star \star *} p<0.001,{ }^{* \star} p<0.0001$. Unpaired, two-tailed, standard t-test). 
a

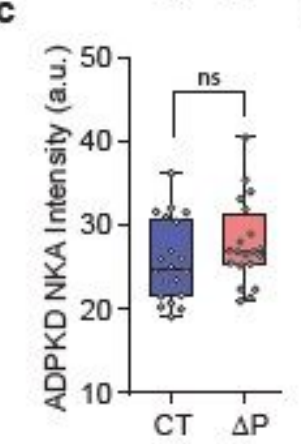

g

Normal tubule

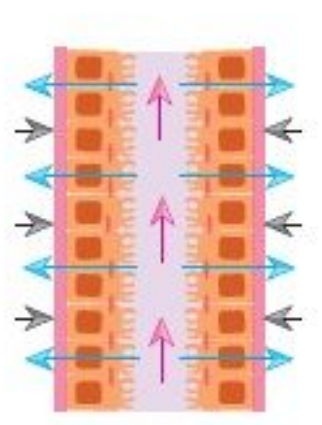

b

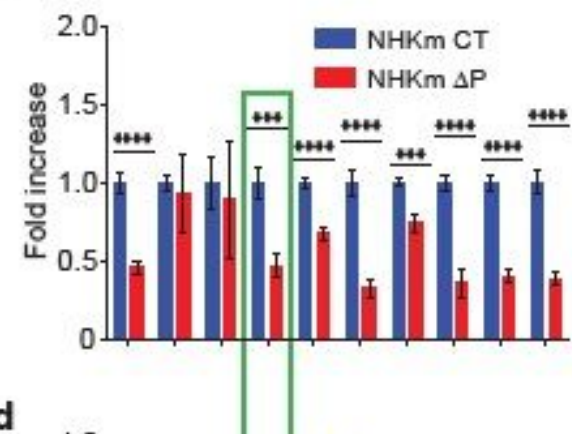

d

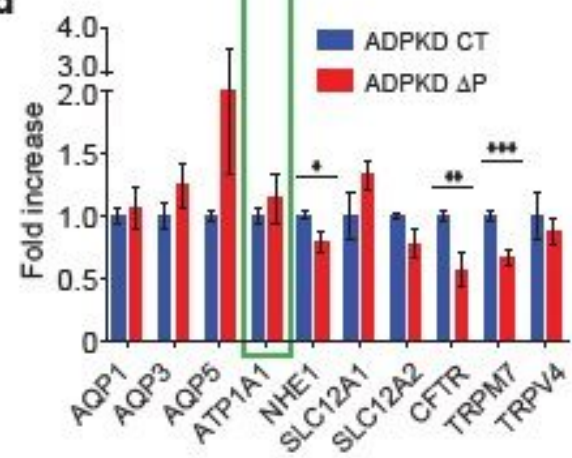

h
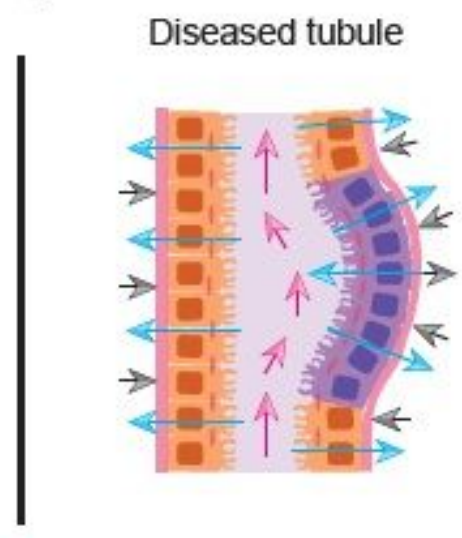

Luminal fluid flow

e

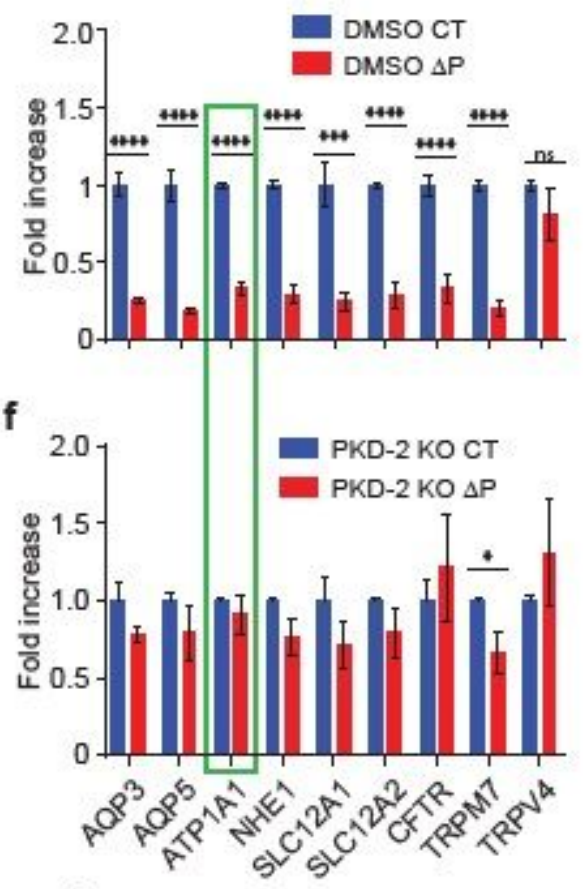

i

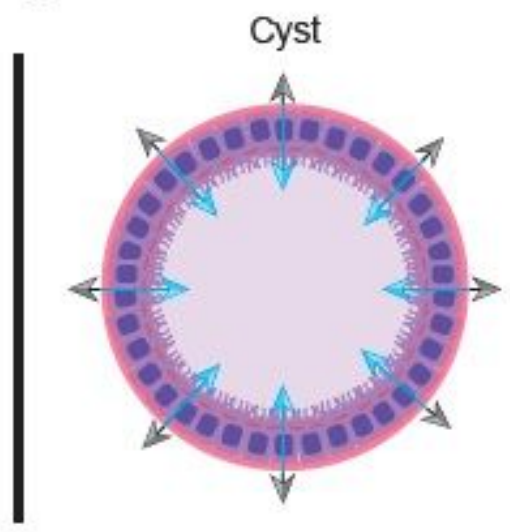

Force $\longrightarrow$

\section{Figure 4}

Diseased cells have defects in sensing hydrostatic pressure as compared to normal cells (a) Comparison of total $\mathrm{Na}+\mathrm{K}+$ ATPase (NKA) expression in human NHKm cells and ADPKD cystic cells using antibody staining of the $\mathrm{Na} / \mathrm{K}$ ATPase a-subunit. Maximum intensity projections of confocal stacks were used to quantify NKA expression. The epithelium was studied under two conditions: CT (Control, DP $=0$ ) and DP (hydrostatic pressure gradient, DP $=200 \mathrm{~Pa}$ for 5 hours). Plot showing total intensity of NKA in NHKc cells at CT and DP. $(n=20, N=3)$. (b) Comparison of relative expression changes of genes in NHKm cells for $C T(D P=0)$ and $D P(D P=200 P a) .(n=9, N=3)$. (c) Plot showing total intensity of NKA in human ADPKD cystic cells for CT (DP $=0)$ and $D P=-200 P a(n s=$ not significant, $p=0.74),(n=20, N=3)$. (d) Comparison of gene expression changes in human ADPKD cystic cells for CT $(D P=0)$ and DP $=-200 P a$ 
$(n=9, N=3)$. Green rectangle indicates the comparison for ATP1A1 (which encodes for NKA). For human NHKm cells, mRNA levels of ATP1A1 decreased significantly (*** $\$<0.001 \$$ ) under hydrostatic pressure for 10 hours but remained unchanged in case of cystic cells $(p=0.5170)$. (e) Comparison of relative expression changes of genes in inducible mouse cells (Pkd2fl/fl, Pax8rtTA, TetOCre) with DMSO vehicle for $C T(D P=0)$ and $D P=200 \mathrm{~Pa}(n=6, N=2)$. (f) Comparison of relative expression changes of genes in doxycycline induced Pkd2 KO mouse cells for CT (DP $=0)$ and $D P=200 \mathrm{~Pa} .(n=12, N=4)$. For normal mouse cells, the mRNA levels of ATP1A1 gene increased significantly $(\star \star \star \star p ~<~ 0.0001)$ under hydrostatic pressure for 10 hours but remained unchanged in case of Pkd2 KO cells $(p=0.4856)$. Error bars indicate SEM. ( ${ }^{\star} p<0.05,{ }^{\star \star} p<0.01,{ }^{\star \star \star} p<0.001,{ }^{* \star *} \mathrm{p}<0.0001$. Unpaired, two-tailed, standard t-test). (g) A schematic representation of a tubular nephron section in a normal kidney. The blue arrows indicate absorptive fluid flux from the lumen into the interstitial space. The black arrows indicate the pumping force generated by cells. The tubular structure is stabilized by the pumping force due to apico-basal fluid flux (radially in). (h) During cyst initiation, cell proliferation can alter the tubule geometry and the local fluid flow pattern, results in a lowered FSS. ADPKD cells may respond by reversing the fluidic pumping direction as shown in Fig. 3o, which also reverses the pumping force that further destabilizes the tubule. (i) In mature cysts, fluid pumping elevates pressure in the cyst and aids in cyst expansion.

\section{Supplementary Files}

This is a list of supplementary files associated with this preprint. Click to download.

- ExtendedDataFig.1.pdf

- ExtendedDataFig.2.pdf

- ExtendedDataFig.3.pdf

- ExtendedDataFig.4.pdf

- ExtendedDataFig.5.pdf

- ExtendedDataFig.6.pdf

- ExtendedDataFig.7.pdf

- ExtendedDataFig.8.pdf

- ExtendedDataFig.9.pdf

- ExtendedDataFig.10.pdf

- ExtendedDataFig.11.pdf

- ExtendedDataFig.12.pdf

- ExtendedDataFig.13.pdf

- SupplementaryVideo1 compressed.avi

- SupplementaryVideo2compressed.avi

- Supplementaryvideo3.mp4 
- SupplementaryVideo5.avi

- Supplementaryvideo4.mp4

- SupplementaryVideo6.avi

- Supplementallnformation.pdf 